\title{
Conical refraction Nd:CALGO laser with dual-wavelength output
}

Akbari, R., Xu, X., Xu, J., Fedorova, K., Sokolovskii, G., et al.

R. Akbari, X. Xu, J. Xu, K. A. Fedorova, G. S. Sokolovskii, E. U. Rafailov, A. Major, "Conical refraction Nd:CALGO laser with dual-wavelength output," Proc. SPIE 11664, Solid State Lasers XXX: Technology and Devices, 1166419 (5 March 2021); doi: 10.1117/12.2582440

SPIE. Event: SPIE LASE, 2021, Online Only 


\title{
Conical refraction Nd:CALGO laser with dual-wavelength output
}

\author{
R. Akbari ${ }^{1}$, X. Xu ${ }^{2}$, J. Xu ${ }^{3}$, K.A. Fedorova ${ }^{4}$, G.S. Sokolovskii ${ }^{5,6}$, E.U. Rafailov ${ }^{7,8}$, and A. Major ${ }^{1}$ \\ ${ }^{1}$ Department of Electrical and Computer Engineering, University of Manitoba, 75A Chancellors Circle, \\ Winnipeg, MB, R3T 5V6, Canada \\ ${ }^{2}$ Jiangsu Key Laboratory of Advanced Laser Materials and Devices, School of Physics and Electronic \\ Engineering, Jiangsu Normal University, 221116 Xuzhou, China \\ ${ }^{3}$ School of Physics Science and Engineering, Institute for Advanced Study, Tongji University, 200092 \\ Shanghai, China \\ ${ }^{4}$ Department of Physics, Philipps-Universität Marburg, Marburg, 35032, Germany \\ ${ }^{5}$ Ioffe Institute, St. Petersburg, 194021, Russia \\ ${ }^{6}$ Saint Petersburg Electrotechnical University (LETI), St. Petersburg, 197022, Russia \\ ${ }^{7}$ School of Engineering \& Applied Science, Aston University, Birmingham, B4 7ET, UK \\ ${ }^{8}$ Saratov State University, Astrakhanskaya Str. 83, Saratov 410012, Russia
}

\begin{abstract}
We demonstrated continuous-wave dual-wavelength operation of a $\mathrm{Nd}$ :CALGO laser with intracavity conerefringent element. The laser produced conically refracted dual-wavelength radiation output with more than $100 \mathrm{~mW}$ of power. Dual-wavelength radiation was generated owing to the broad gain bandwidth of the Nd:CALGO crystal.
\end{abstract}

Keywords: Conical refraction, $\mathrm{Nd}$-ion lasers, diode-pumped lasers.

\section{INTRODUCTION}

Conical refraction (CR) laser beams have attracted a lot of attention due to their potential applications for communication, metrology, microscopy, optical trapping, etc. [1-4]. In CR a biaxial crystal transforms the propagating Gaussian beam into a hollow cylinder with an annular transverse intensity profile [5-12]. The unique property of the CR laser beam is that the polarization state distribution along with the CR pattern results in every two diametrically opposite points to have orthogonal polarizations. At the same time CR light beam exhibits symmetrical evolution pattern with respect to the so-called Lloyd image plane and evolves into a bright center spot (i.e. the Raman spot) in the far-field. The generation of the CR laser beams directly from the laser oscillators has been also demonstrated where single wavelength radiation was produced [12-19]. Some applications, however, would benefit from multiple wavelengths. Multi-wavelength operation was demonstrated from a number of gain media [20-25].

Recently, we have demonstrated a CR laser based on separate intracavity conerefringent element (CRE) and laser host (Nd:YVO), and CR laser beam with unprecedented quality with fully resolved concentric rings was generated [13]. The separation of the CR element and the laser host made it possible to alleviate the complex pumping and facilitate the power scalability of the laser [26,27]. The laser properties such as wavelength was also independent from the CRE material.

In this work we demonstrate generation of dual-wavelength (DW) CR radiation directly from a single laser. Our approach was based on a gain medium with broadband gain and intracavity conerefringent element (CRE). Among the Nd-doped laser crystals, $\mathrm{Nd}$ :CALGO $\left(\mathrm{Nd}: \mathrm{CaGdAlO}_{4}\right)$ exhibits a broad emission bandwidth $(\sim 15 \mathrm{~nm})$ with a strong absorption line at $806.5 \mathrm{~nm}$ and the largest emission cross-sections at $1067.7 \mathrm{~nm}(\mathrm{E} / / \pi)$ and $1078.8 \mathrm{~nm}(\mathrm{E} / / \sigma)$ [28-31]. The broad gain bandwidth of the crystal makes it a suitable candidate for wide wavelength tuning range, as well as multi-wavelength laser operation [30,31]. The demonstrated laser produced DW radiation at $1078.6 \mathrm{~nm}$ and 1080.8 $\mathrm{nm}$. The laser delivered CR laser beam output with excellent quality and output power of $>100 \mathrm{~mW}$. Local polarization states of the produced beam were analyzed with a polarizer and its CR nature was confirmed. 


\section{EXPERIMENTAL SETUP}

A $5 \mathrm{~mm}$-long 1 at.\% (a-cut) Nd:CALGO crystal was used as the gain medium and an $18 \mathrm{~mm}$-long KGW crystal served as a CRE. The biaxial crystal of KGW is well-known for its strong optical anisotropy and good laser properties [3237]. Both crystals were placed in a modified delta-cavity with additional focusing mirror for the CRE (see figure 1). The laser was pumped by a home-built Ti:sapphire laser. The wavelength of the pump was adjusted at $806.5 \mathrm{~nm}$ by using a birefringent filter plate and an output power of $750 \mathrm{~mW}$ could be generated. The pump beam was focused to a spot size of around $35 \mu \mathrm{m}$ in the Nd:CALGO crystal.

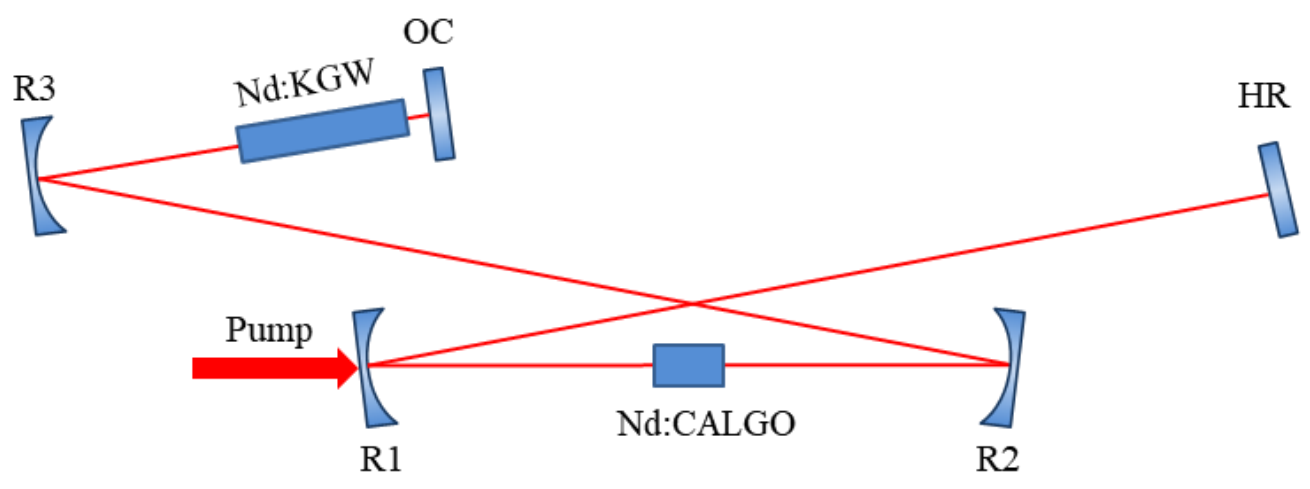

Figure 1. Schematic diagram of the laser cavity. R1=R2=-100 mm, R3=-150 mm. OC: output coupler, HR: highly reflective mirror. $5 \%$ output coupling was used.

\section{RESULTS AND DISCUSSION}

The laser was initially aligned for free-running operation without the CRE crystal in the cavity and modified R3-OC separation. The laser was naturally oscillating with two spectral lines at $1078.7 \mathrm{~nm}$ and $1079.6 \mathrm{~nm}$ (see figure 2). The maximum output power of $140 \mathrm{~mW}$ under $750 \mathrm{~mW}$ of pump power could be generated. The polarization state of the laser output was determined to be horizontal $(\mathrm{E} / / \sigma)$.

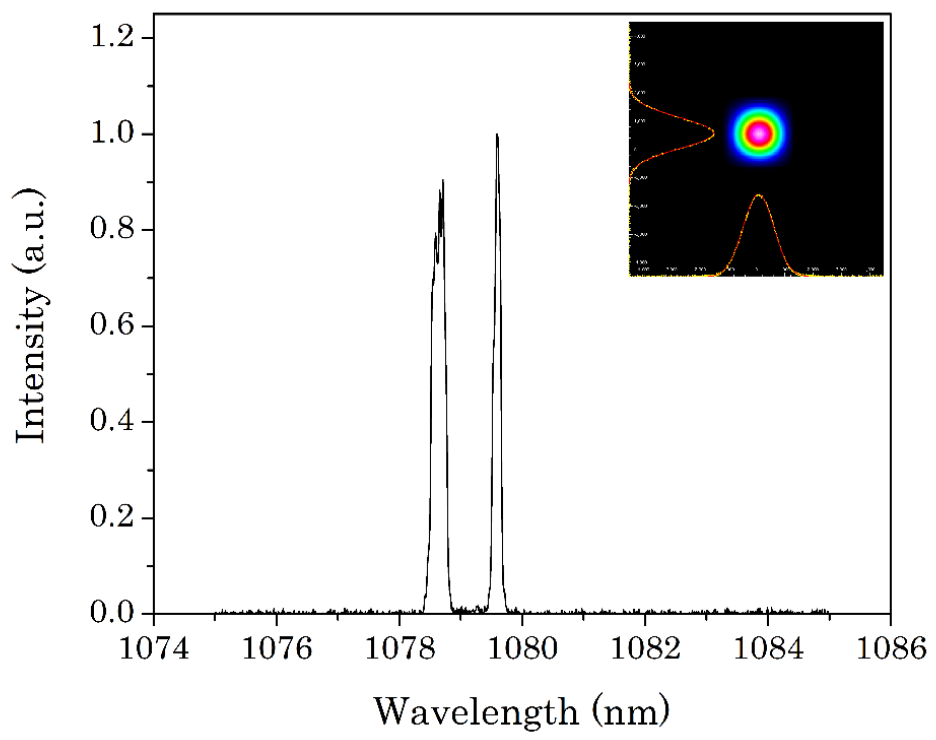

Figure 2. Spectrum of the free-running laser (i.e. without the CRE element in the cavity). Inset shows intensity profile of the output laser beam. 
In order to generate CR laser output radiation, the CRE was placed between the focusing mirror R3 and the OC with increased R3-OC separation (see figure 1). In the next step, the CRE had to be aligned with respect to the propagation axis of the laser mode. For large misalignment between the optical axis (OA) of the CRE and the axis of the laser mode, the laser produced a single spot beam with negligible power. By approaching the parallel direction of the CRE and the axis of the laser mode, the laser output resembled a double refraction mode or a single spot and a crescent with an increased power. At this stage, fine adjustment of the CRE and the output coupler was required to obtain a laser beam with a fully resolved CR pattern (see figure 3(a)). The produced beam was imaged with a single lens onto a CCD camera [13]. The position of the image plane was calculated to be around $3 \mathrm{~mm}$ outside of the output coupler. The evolution of the CR laser radiation in free-space was examined by moving the camera along the axis of propagation (see figure 3(b)-(d)). Similar to the traditional CR beam, the generated laser beam evolved from the Lloyd image plane to the Raman spot. The local polarization state along the CR ring was also investigated by using a rotating polarizer and orthogonal polarization for diametrically opposite point along the ring was confirmed (see figure 3(e)).
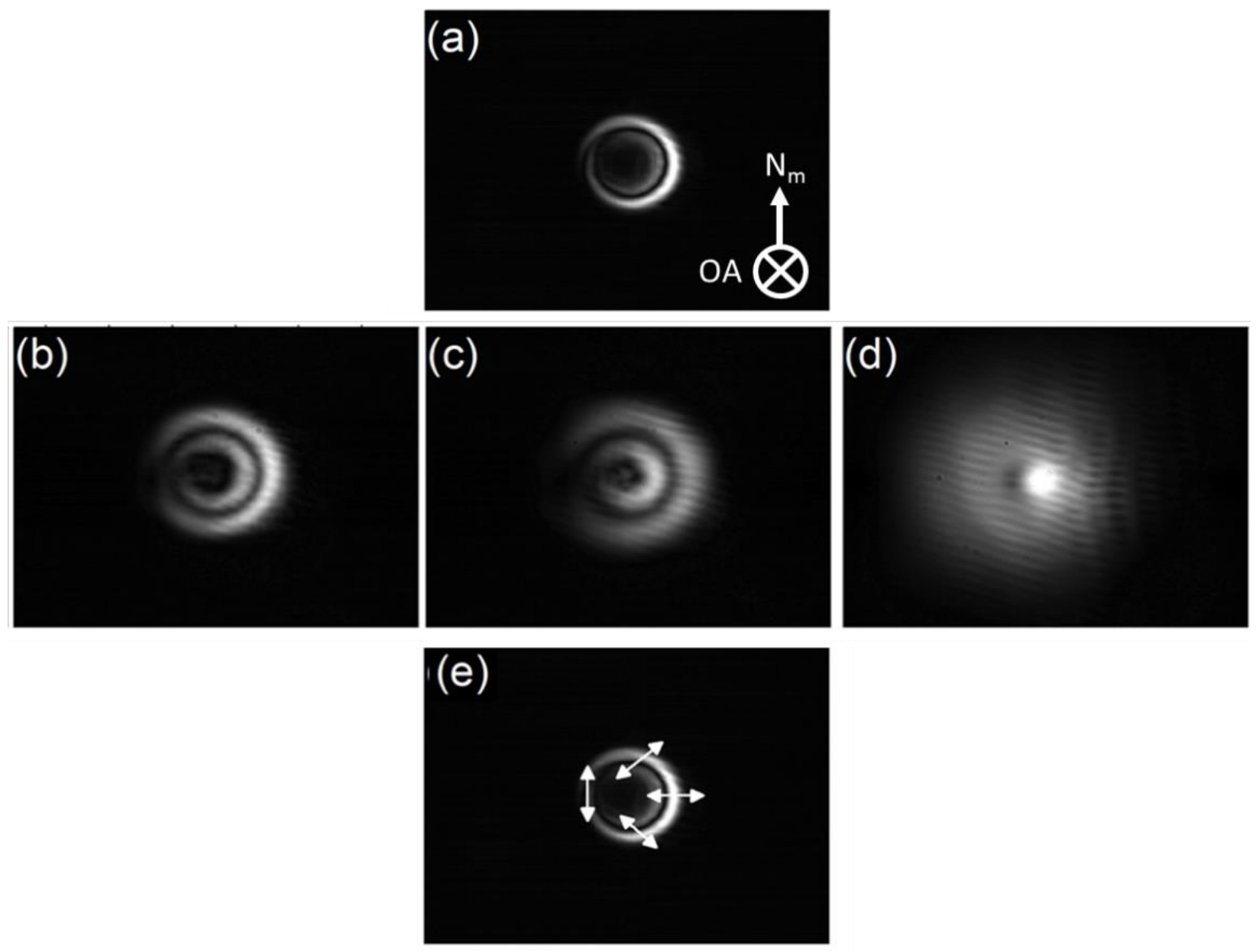

Figure 3. The CR laser beam at (a) Lloyd image plane and (b) its free-space evolution (b)-(d) for the vertical orientation of the $\mathrm{N}_{\mathrm{m}}$-axis of the CRE, (e) the local polarization states along the CR beam.

The wavelength of the produced CR laser is shown in figure 4. DW oscillation at $1078.62 \mathrm{~nm}$ and $1080.77 \mathrm{~nm}$ was obtained. It should be noted that the wavelength of the CR laser was sensitive to the alignment of the CRE crystal. At the same time, the formation of CR beam is generally a function of wavelength of the propagating beam via refractive index dependence on wavelength [38]. Therefore, it was tried to achieve a resolvable CR ring while maintaining a 
DW nature of the laser by simultaneous alignment of the CRE and OC. Further attempts to equalize the spectral intensity of the oscillating wavelength resulted in lower resolvability of the CR rings.

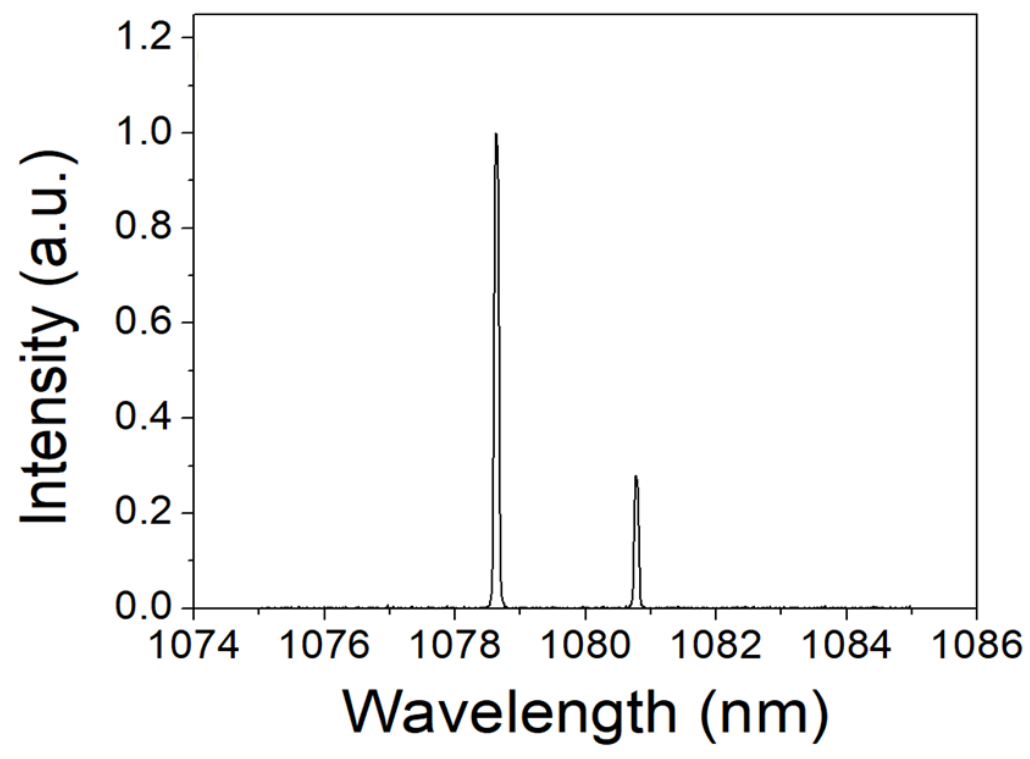

Figure 4. The spectrum of the DW CR laser.

The DW CR laser could deliver up to $121 \mathrm{~mW}$ of output power at $750 \mathrm{~mW}$ of pump power. This corresponded to $16 \%$ optical-to-optical efficiency. The DW CR laser performance for different pump power was also examined where slope efficiency of around $17 \%$ was obtained (see figure 5).

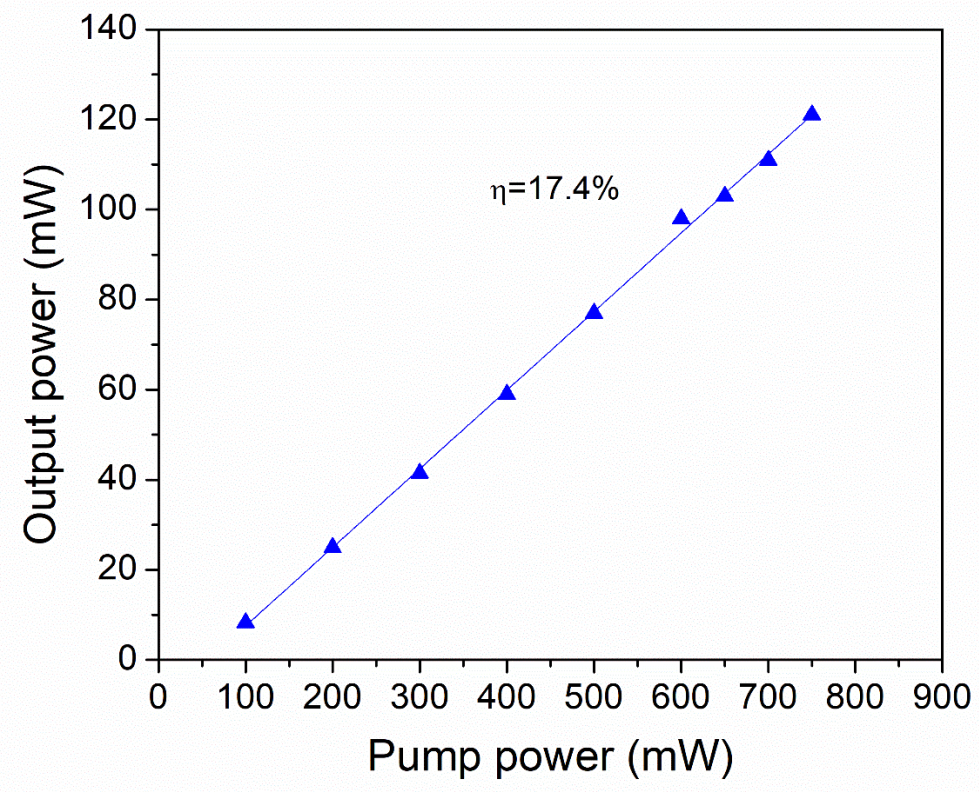

Figure 5. Output power of DW CR laser vs. incident pump power. 


\section{CONCLUSIONS}

Dual-wavelength operation of a CR laser based on broadband laser host (Nd:CALGO) and an intracavity conerefringent crystal was demonstrated. The laser delivered fully resolved CR beam with more than $100 \mathrm{~mW}$ of power at $1078.6 \mathrm{~nm}$ and $1080.8 \mathrm{~nm}$. The generated CR beam exhibited similar propagation evolution from the Lloyd image plane into the bright Raman spot. Orthogonal polarization states of radially opposite points along the CR ring were also confirmed. DW nature of the generated laser radiation was due to the use of a broad-bandwidth laser gain medium. On the other hand, generation of the CR laser radiation with independent CRE enabled us to separate the laser properties from the CR beam formation. As a future work, it is possible to make the spectral intensity of the oscillating wavelengths equal and tune the wavelength of the laser by using a birefringent filter plate (BRF) [31,3945] or dual-crystal arrangement [46]. BRFs exhibit wavelength-dependent transmission loss that can enable one to impose higher loss on spectral regions where there is high level of gain. By exploiting this feature, the oscillating wavelength with higher spectral intensity can be further suppressed so that DW components with equal intensities can be generated. In a similar fashion, the wavelength of the CR laser radiation can be also tuned over the available gain bandwidth of laser materials [47-51].

\section{ACKNOWLEDGEMENTS}

The authors would like to acknowledge funding of this project provided by the Natural Sciences and Engineering Research Council of Canada (NSERC); University of Manitoba (U of M); Canadian Foundation for Innovation (CFI).

\section{REFERENCES}

[1] J. Caron, C. Fallet, J.-Y. Tinevez, L. Moisan, L. P. (Ori) Braitbart, G. Y. Sirat, and S. L. Shorte, "Conical diffraction illumination opens the way for low phototoxicity super-resolution imaging," Cell Adh. Migr. 8, 430-439 (2014).

[2] S. Rosen, G. Y. Sirat, H. Ilan, and A. J. Agranat, "A sub wavelength localization scheme in optical imaging using conical diffraction," Opt. Express 21, 10133-10138 (2013).

[3] D. P. O'Dwyer, K. E. Ballantine, C. F. Phelan, J. G. Lunney, and J. F. Donegan, "Optical trapping using cascade conical refraction of light," Opt. Express 20, 21119-21125 (2012).

[4] A. Turpin, Y. Loiko, T. K. Kalkandjiev, and J. Mompart, "Free-space optical polarization demultiplexing and multiplexing by means of conical refraction," Opt. Lett. 37, 4197 (2012).

[5] A. Turpin, Y. V. Loiko, T. K. Kalkandjiev, and J. Mompart, "Conical refraction: fundamentals and applications," Laser Photonics Rev. 10, 750 (2016).

[6] A. M. Belsky and A. P. Khapalyuk, "Internal conical refraction of bounded light beams in biaxial crystals," Opt. Spectrosc. 44, 436 (1978).

[7] A. M. Belsky and M. A. Stepanov, "Internal conical refraction of coherent light beams," Opt. Commun. 167, 1 (1999).

[8] M. V. Berry, "Conical diffraction asymptotics: fine structure of Poggendorff rings and axial spike," J. Opt. A 6, 289 (2004).

[9] C. F. Phelan, K. E. Ballantine, P. R. Eastham, J. F. Donegan, and J. G. Lunney, "Conical diffraction of a Gaussian beam with a two crystal cascade," Opt. Express 20, 13201 (2012).

[10] G. S. Sokolovskii, D. J. Carnegie, T. K. Kalkandjiev, and E. U. Rafailov, "Conical Refraction: New observations and a dual cone model," Opt. Express 21, 11125 (2013).

[11] A. Turpin, Y. V. Loiko, T. K. Kalkandkiev, H. Tomizawa, and J. Mompart, "Super-Gaussian conical refraction beam," Opt. Lett. 39, 4349 (2014).

[12] M. V. Berry, M. R. Jeffrey, and J. G. Lunney, "Conical diffraction: observations and theory," Proc. R. Soc. A 462, 1629 (2006).

[13] R. Akbari, C. Howlader, K. A. Fedorova, G. S. Sokolovskii, E. U. Rafailov, and A. Major, "Conical refraction output from a Nd: $\mathrm{YVO}_{4}$ laser with an intracavity conerefringent element," Opt. Lett. 44(3), 642-645 (2019).

[14] J. Hellström, H. Henricsson, V. Pasiskevicius, U. Bünting, and D. Haussmann, "Polarization-tunable Yb: KGW laser based on internal conical refraction," Opt. Lett. 32, 2783 (2007). 
[15] A. Brenier, "Revealing modes from controlling an off-optical axis conical diffraction laser," Laser Phys. 27, 105001 (2017).

[16] A. Abdolvand, K. G. Wilcox, T. K. Kalkandjiev, and E. U. Rafailov, "Conical refraction Nd: $\mathrm{KGd}_{\left(\mathrm{WO}_{4}\right)_{2}}$ laser," Opt. Express 18, 2753 (2010).

[17] A. Brenier, "Lasing with conical diffraction feature in the $\mathrm{KGd}\left(\mathrm{WO}_{4}\right)_{2}$ : $\mathrm{Nd}$ biaxial crystal,"Appl. Phys. B 122, 237 (2016).

[18] K. G. Wilcox, A. Abdolvand, T. K. Kalkandjiev, and E. U. Rafailov, "Laser with simultaneous Gaussian and conical refraction outputs," Appl. Phys. B 99, 619 (2010).

[19] R. Cattoor, I. Manek-Hönninger, D. Rytz, L. Canioni, and M. Eichhorn, "Laser action along and near the optic axis of a holmium-doped KY(WO$)_{2}$ crystal," Opt. Lett. 39, 6407 (2014).

[20] T. Waritanant, A. Major, "Diode-pumped Nd: $\mathrm{YVO}_{4}$ laser with discrete multi-wavelength tunability and high efficiency," Opt. Lett. 42, 1149-1152 (2017).

[21] T. Waritanant, A. Major, "Discretely selectable multiwavelength operation of a semiconductor saturable absorber mirror mode-locked Nd:YVO 4 laser," Opt. Lett. 42, 3331-3334 (2017).

[22] T. Waritanant, A. Major, "Dual-wavelength operation of a diode-pumped Nd:YVO ${ }_{4}$ laser at the 1064.1 \& $1073.1 \mathrm{~nm}$ and $1064.1 \& 1085.3 \mathrm{~nm}$ wavelength pairs," Appl. Phys. B 124(5), 87 (2018).

[23] M. Nadimi, T. Waritanant, A. Major, "Discrete multi-wavelength tuning of a continuous wave diode-pumped Nd:GdVO 4 laser," Laser Phys. Lett. 15(5), 055002 (2018).

[24] M. Nadimi, A. Major, "Continuous-wave dual-wavelength operation of a diode-pumped Nd:GdVO 4 laser at the 1063 \& $1071 \mathrm{~nm}, 1063$ \& $1083 \mathrm{~nm}$ and 1083 \& $1086 \mathrm{~nm}$ wavelength pairs," Laser Phys. 28(9), 095001 (2018).

[25] S. Ghanbari and A. Major, "High power continuous-wave dual-wavelength alexandrite laser," Laser Phys. Lett. 14(10), 105001 (2017).

[26] C. Howlader, M. Nadimi, T. Waritanant, K.A. Fedorova, G.S. Sokolovskii, E.U. Rafailov, A. Major, "High power Nd:YVO-KGW conical refraction laser," Proc. SPIE 10904, 109041X (2019).

[27] Md. A. R. Reza, R. Akbari, K. A. Fedorova, G. S. Sokolovskii, E. U. Rafailov, and A. Major, "Diode-pumped $\mathrm{Yb}:$ CALGO laser with conical refraction output," Proc. SPIE 11259, 112590G (2020).

[28] A. A. Lagatskii, N. V Kuleshov, V. G. Shcherbitskii, V. F. Kleptsyn, V. P. Mikhailov, V. G. Ostroumov, and G. Huber, "Lasing characteristics of a diode-pumped $\mathrm{Nd}^{3+}: \mathrm{CaGdAlO}_{4}$ crystal," Quantum Electron. 27, 1517 (1997).

[29] J. Di, X. Sun, X. Xu, C. Xia, Q. Sai, H. Yu, Y. Wang, L. Zhu, Y. Gao, and X. Guo, "Growth and spectral characters of Nd:CaGdAlO 4 crystal," EPJ Appl. Phys. 74, 1-6 (2016).

[30] P. Loiko, J.M. Serres, X. Mateos, X. Xu, J. Xu, U. Griebner, V. Petrov, M. Aguiló, F. Díaz, A. Major, "Dualwavelength Nd:CaLnAlO 4 lasers at $1.365 \& 1.390 \mu \mathrm{m}$," Proc. SPIE 10511, 105111V (2018).

[31] R. Akbari, P. Loiko, J. Xu, X. Xu, A. Major, "Dual-wavelength Nd:CALGO laser based on an intracavity birefringent filter," Proc. SPIE 10896, 1089611 (2019).

[32] C. McDonald, C. McDougall, E. Rafailov, and D. McGloin, "Characterizing conical refraction optical tweezers," Opt. Lett. 39, 6691 (2014).

[33] I. V. Mochalov, "Laser and nonlinear properties of potassium gadolinium tungstate laser crystal: $\mathrm{KGd}\left(\mathrm{WO}_{4}\right)_{2} \mathrm{Nd}^{3+}(\mathrm{KGW}: \mathrm{Nd})$, , Opt. Eng. 36, 1660 (1997).

[34] R. C. Talukder, M. Z. E. Halim, T. Waritanant, and A. Major, "Multiwatt continuous wave Nd:KGW laser with hot-band diode pumping," Opt. Lett. 41, 3810 (2016).

[35] M. Z. E. Halim, R. C. Talukder, T. Waritanant, and A. Major, "Passive mode locking of a Nd:KGW laser with hot-band diode pumping," Laser Phys. Lett. 13, 105003 (2016).

[36] H. Zhao, A. Major, "A continuous wave Yb:KGW laser with polarization-independent pump absorption," Laser Phys. 23, 095001 (2013).

[37] A. Major, N. Langford, T. Graf and A. I. Ferguson, "Additive-pulse mode locking of a diode-pumped $\mathrm{Nd}: \mathrm{KGd}\left(\mathrm{WO}_{4}\right)_{2}$ laser,” Appl. Phys. B 75(4-5), 467-469 (2002).

[38] J. P. Fève, B. Boulanger, and G. Marnier, "Experimental study of internal and external conical refractions in KTP,” Opt. Commun. 105(3-4), 243-252 (1994).

[39] R. Akbari, H. Zhao, and A. Major, "High-power continuous-wave dual-wavelength operation of a diodepumped Yb:KGW laser," Opt. Lett. 41, 1601-1604 (2016).

[40] S. Manjooran, P. Loiko, and A. Major, "A discretely tunable dual-wavelength multi-watt Yb:CALGO laser," Appl. Phys. B 124, 13 (2018). 
[41] R. Akbari and A. Major, "Generation of THz frequency offset with dual-wavelength Yb:KGW laser," Proc. SPIE 11259, 112591U (2020).

[42] Md. A. R. Reza, R. Akbari, and A. Major "Dual-wavelength Yb:CALGO laser with wavelength spacing tunability," Proc. SPIE 11259, 112591S (2020).

[43] U. Demirbas, "Off-surface optic axis birefringent filters for smooth tuning of broadband lasers," Appl. Opt. 56, 7815-7825 (2017).

[44] U. Demirbas, R. Uecker, J. G. Fujimoto, and A. Leitenstorfer, "Multicolor lasers using birefringent filters: experimental demonstration with Cr:Nd:GSGG and Cr:LiSAF," Opt. Express 25, 2594 (2017).

[45] E. Beyatli and U. Demirbas, "Widely tunable dual-wavelength operation of Tm:YLF, Tm:LuAG, and Tm:YAG lasers using off-surface optic axis birefringent filters," Appl. Opt. 57, 6679 (2018).

[46] M. Nadimi, C. Onyenekwu, A. Major, "Continuous-wave dual-wavelength operation of the in-band diodepumped Nd:GdVO $/ \mathrm{Nd}: \mathrm{YVO}_{4}$ composite laser with controllable spectral power ratio," Appl. Phys. B 126(5), 75 (2020).

[47] S. Manjooran, A. Major, "Efficient operation of a diode-pumped multi-watt continuous wave Yb:KYW laser with excellent beam quality," Laser Phys. Lett. 14(3), 035805 (2017).

[48] S. Manjooran and A. Major, "Diode-pumped $45 \mathrm{fs}$ Yb:CALGO laser oscillator with $1.7 \mathrm{MW}$ of peak power," Opt. Lett. 43(10), 2324-2327 (2018).

[49] M. Nadimi, T. Waritanant, and A. Major, "High power and beam quality continuous-wave Nd:GdVO 4 laser in-band diode-pumped at $912 \mathrm{~nm}$," Photon. Res. 5(4), 346-349 (2017).

[50] M. Kowalczyk, A. Major, and J. Sotor, "High peak power ultrafast $\mathrm{Yb}: \mathrm{CaF}_{2}$ oscillator pumped by a singlemode fiber-coupled laser diode," Opt. Express 25(21), 26289-26295 (2017).

[51] S. Ghanbari, K.A. Fedorova, A.B. Krysa, E.U. Rafailov, A. Major, "Femtosecond Alexandrite laser passively mode-locked by InP/InGaP quantum-dot saturable absorber,” Opt. Lett. 43(2), 232-234 (2018). 